\title{
Using submerged fermentation to fast increase N6-(2-hydroxyethyl)-adenosine, adenosine and polysaccharide productions of Cordyceps cicadae NTTU 868
}

\author{
Bo-Jun Ke and Chun-Lin Lee
}

\begin{abstract}
Cordyceps cicadae is a well-known traditional Chinese medicine for treating palpitations and eye diseases. It contains several bioactive compounds such as adenosine, N6-(2-hydroxyethyl)-adenosine (HEA), and polysaccharide. Those bioactive compounds have been reported to perform anti-oxidation and anti-inflammatory properties and provide renal protection. In this study, we researched different fermentation conditions in order to enhance the biomass, adenosine, HEA, and polysaccharide productions of C. cicadae NTTU 868. Solid fermentation was carried out with different grain substrates (barley, oat, rice and wheat). Various submerged fermentation scales were used to produce the C. cicadae NTTU 868 mycelium. The results of solid fermentation revealed that C. cicadae NTTU 868 produced higher adenosine and HEA concentrations in oat rather than in other substrates. C. cicadae NTTU 868 mycelium had obtained the highest concentrations of adenosine and HEA on Day 2 as using the small-scale submerged fermentation. Furthermore, potato dextrose broth with extra $0.2 \%$ of yeast extract was able to result in higher HEA concentration. In conclusion, using submerged fermentation to culture C. cicadae NTTU 868 resulted in more efficient adenosine, HEA, and polysaccharide productions than using solid-fermentation, especially when $0.2 \%$ of yeast extract was used in the PDB. Importantly, this can be easily scaled-up in the fermentation industry.
\end{abstract}

Keywords: Cordyceps cicadae NTTU 868, Adenosine, N6-(2-hydroxyethyl)-adenosine (HEA), Polysaccharide

\section{Introduction}

Cordyceps species are used in many pharmacological treatments including to treat cancer (Lee et al. 2006; Park et al. 2005), anti-oxidation (Yu et al. 2006), antiinflammatory treatments (Lu et al. 2015; Won and Park 2005), anti-aging (Ramesh et al. 2012), immunopotentiation (Weng et al. 2002; Zhou et al. 2008) and hyperlipidemia (Sohn et al. 2012). However, Cordycpes cicadae is a well-known source of traditional Chinese Cordycpes medicine in Asia. The Cicada flammata worm and other insects are infected by fungi $C$. cicadae in winter. The fruit bodies grow on the pupa of the worm in summer. The length is about 0.1 to $4 \mathrm{~cm}$. The wild C. cicadae

*Correspondence: cllee@nttu.edu.tw

Department of Life Science, National Taitung University, 369, Sec. 2, University Rd., Taitung 95092, Taiwan, ROC grows in high humid conditions on mountains between 80 and $500 \mathrm{~m}$ high in Asia. Previous studies indicated that $C$. cicadae produced many bioactive compounds, such as adenosine (Wang et al. 2012; Zeng et al. 2014), N6-(2-hydroxyethyl)-adenosine (HEA) (Meng et al. 2015), ergosterol (Weng et al. 2002; Zhu et al. 2014), and polysaccharides (Sharma et al. 2018). In addition, HEA, one of the characteristic bioactive compounds in C. cica$d a e$, has been proven to perform renal protective qualities, and anti-inflammatory property (Lu et al. 2015). C. cicadae-produced polysaccharide can inhibit the levels of reactive oxygen species (ROS) and exhibited the activities of anti-oxidative enzymes (Peng et al. 2013). Adenosine, a purine nucleoside also has anti-inflammatory and antihepatic fibrosis qualities and prevents diabetes (Koupenova and Ravid 2013). 
Cordyceps cicadae fruit body has been harvested successfully produced using artificial solid culture but the culture is more than 50 days (Wang et al. 2014). To increase the functional compounds production and short the culture time were important topic for the development of C. cicadae. Submerged culture was another culture technology for the mycelium of $C$. cicadae. In our previous study, the submerged culture product of $C$. cicadae has been proven to prevent the development of liver-fibrosis in mice induced by $\mathrm{CCl}_{4}$ injection. Furthermore, HEA, adenosine, and polysaccharides produced by submerged culture were proven as the functional components for liver protection (Ke and Lee 2018). Therefore, submerged culture may be the novel culture to instead of solid fermentation for producing HEA and polysaccharides.

In this study, we researched different culture methods and conditions to short the culture time and produce high adenosine, HEA, and polysaccharide contents in C. cicadae NTTU 868. Furthermore, we also conducted pilot-up fermentation to create medical potential for the fermentation industry.

\section{Materials and methods Chemicals}

99\% purity of adenosine (Sigma-Aldrich Co., St. Louis, Missouri, USA) and 95\% purity of HEA (Ark Pharm, Inc., Libertyville, Illinois, USA) were used as standard in the HPLC test.

\section{Isolation and culture}

Cordyceps cicadae NTTU 868 (C. cicadae BCRC 930197) deposited in a Bioresource Collection and Resource Center (BCRC, Hsinchu, Taiwan) was incubated in potato dextrose agar (PDA) plates at $24{ }^{\circ} \mathrm{C}$. Sub-culturing was done at 30-days intervals to maintain the viability. The seed culture was cultured in potato dextrose broth (PDB) using a 3-baffles Hinton flask with $100 \mathrm{rpm}$ at $24{ }^{\circ} \mathrm{C}$ for $72 \mathrm{~h}$.

\section{Solid fermentation}

Barley, oat, rice and wheat were used as grain substrates. $100 \mathrm{~g}$ of grain substrates were mixed with PDA in plastic boxes, which had 14 cotton balls acting as an air tunnel which were sterilized in an autoclave at $121{ }^{\circ} \mathrm{C}, 1.5 \mathrm{~atm}$ pressure for $30 \mathrm{~min}$. $10 \%$ of the seed-cultured solution was added into the grain substrates. In the first 10 days, the solid fermentation was incubated at $24{ }^{\circ} \mathrm{C}$ without light irradiation to let the mycelium grow in the grain substrates. 10 days later, mycelium covered the grain substrates entirely and the base of the fruit body appeared on the surface. Then the solid fermentation was incubated at $20{ }^{\circ} \mathrm{C}$ for 50 days with light irradiation to irritate fruit body production. The $C$. cicadae NTTU 868 fruit bodies were collected and dried at $55^{\circ} \mathrm{C}$.

\section{Submerged fermentation}

On alternate days submerged fermentation was cultured in PDB with a $10 \%$ seed-cultured solution using a 3-baffles shake flask at $24{ }^{\circ} \mathrm{C}$, shaking at $100 \mathrm{rpm}$ on the 2nd, 4th, 6th, 8th, 10th and 12th day. $5 \%$ of different grain powders (barley, oat or rice) also were used in the submerged fermentation and incubated for 2 and then 10 days. Furthermore, carbon (dextrose, mannitol, or sucrose), nitrogen (monosodium glutamate, peptone or yeast extract) and mineral (potassium chloride, magnesium chloride or ferric chloride) supplements were also used in the submerged fermentation and incubated for 10 days. C. cicadae NTTU 868 mycelium was collected and lyophilized.

\section{Pilot-up submerged fermentation}

The 2-L bottle including 1.5-L of PDB with an additional $0.2 \%$ of yeast extract was designed and manufactured to be a fermentor with a culture condition of $24{ }^{\circ} \mathrm{C}$ and $1.5 \mathrm{v} / \mathrm{v} / \mathrm{m}$ aeration. The C. cicadae NTTU 868 mycelium was sampled once every 2 days and then lyophilized for analysis.

\section{Extraction and quantitative analysis of adenosine and HEA} To extract adenosine, and HEA, C. cicadae NTTU 868 fruit body and mycelium were incubated with 10 volumes of $20 \%$ methanol at $60{ }^{\circ} \mathrm{C}$ for $30 \mathrm{~min}$ and vortexed at every 10 min intervals. Then, we collected the C. cicadae NTTU 868 fruit body and mycelium extract by centrifugation at $15,000 \mathrm{rpm}$ for $10 \mathrm{~min}$. Next, the extraction was incubated at $-20{ }^{\circ} \mathrm{C}$ overnight and then centrifuged at $15,000 \mathrm{rpm}$ for $10 \mathrm{~min}$ to remove polysaccharide (Hung et al. 2015). The supernatant was collected.

Adenosine and HEA were determined by high performance liquid chromatography (HPLC) with a reverse-phase column (Mightysil RP-18 GP $5 \mu \mathrm{m} \mathrm{C18,}$ 250-4.6 mm, Kanto Chemical Co., Inc., Tokyo, Japan) and diode array detector (DAD, L-2000 series, Hitachi, Japan). The mobile phase (A solvent: methanol; B solvent: water) was eluted with $0.8 \mathrm{~mL} / \mathrm{min}$ of flow rate and gradient condition (A solvent: methanol; B solvent: water; 0-3 min, $10 \%$ A; $4-8$ min, $0 \%$ A; $8-18$ min, $0 \%$ A to $100 \%$ A; $18-25 \mathrm{~min}, 10 \%$ A, 28 to $30 \mathrm{~min}$ ). $20 \mu \mathrm{L}$ sample was injected each time. Absorption spectra of eluted compounds were recorded at $262 \mathrm{~nm}$ (Lu et al. 2015).

\section{Extraction and quantitative analysis of polysaccharide Cordyceps cicadae NTTU 868 polysaccharide were} extracted by 10 volumes of ultra-pure water at $95{ }^{\circ} \mathrm{C}$ 
for $60 \mathrm{~min}$ and vortexed at $10 \mathrm{~min}$ intervals. The supernatant was collected by centrifugation at $15,000 \mathrm{rpm}$ for $10 \mathrm{~min}$. The extract was mixed with 5 volumes of $95 \%$ ethanol and then incubated at $4{ }^{\circ} \mathrm{C}$ overnight. The extract was then centrifuged at $15,000 \mathrm{rpm}$ for $10 \mathrm{~min}$ and collected using insoluble precipitate (polysaccharide). To quantify the concentration of polysaccharide, we conducted phenol-sulfuric acid assay.

\section{Statistics}

The data is presented as the mean $\pm \mathrm{SD}$. The means followed by a different letter within each column have significant difference from each other (one-way analysis of variance (ANOVA) with Duncan's multiple rang test, $p<0.05)$.

\section{Results}

Effect of solid-fermentation with different grain substrates on the biomass and adenosine, HEA and polysaccharide concentration of C. cicadae NTTU 868 fruit bodies

In this study, we evaluated the effect of different grain substrates on the production of fruit bodies and their bioactive compounds. The result of fruit bodies production was shown in Fig. 1. Using barley, oat, and rice as the substrates had higher production of fruit bodies than using wheat. C. cicadae NTTU 868 fruit bodies produced significantly higher adenosine concentration under oat substrate rather than under wheat substrate $(p<0.05)$ (Table 1). The C. cicadae NTTU 868 fruit bodies also produced significantly higher HEA concentration under barley, oat, and wheat substrates rather than under rice $(p<0.05)$. However, various grain substrates did not

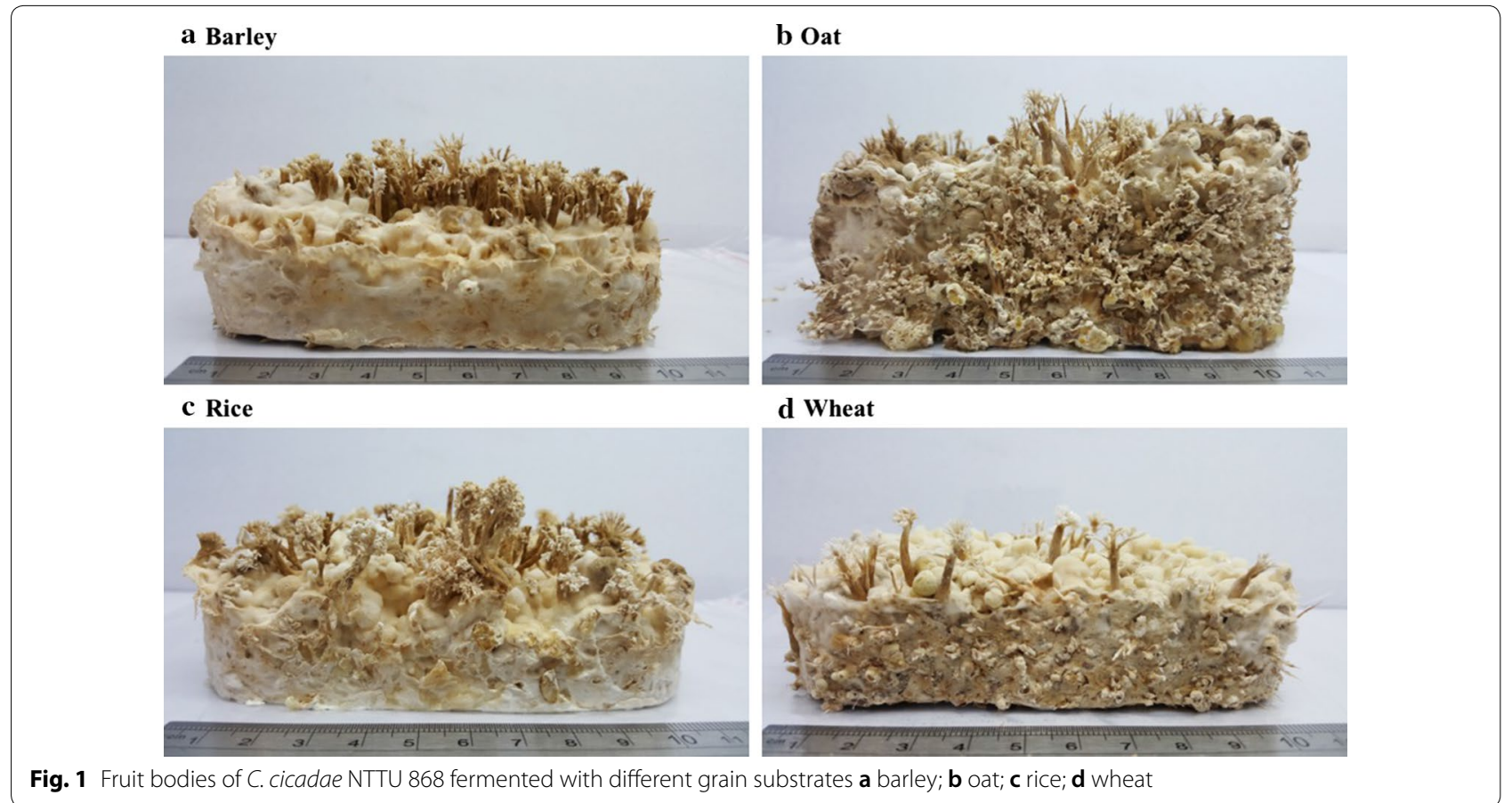

Table 1 The biomass and adenosine, HEA and polysaccharide concentration in solid-fermented C. cicadae NTTU 868 fruit bodies with different grain substrates

\begin{tabular}{lccc}
\hline Substrates & Biomass $(\mathbf{g})$ & Adenosine (mg/kg biomass) & HEA (mg/kg biomass) \\
\hline Barley & $2.56 \pm 1.63^{\mathrm{b}}$ & $893.5 \pm 282.2^{\mathrm{ab}}$ & $930.7 \pm 24.5^{\mathrm{b}}$ \\
Oat & $4.53 \pm 1.93^{\mathrm{bc}}$ & $1176.9 \pm 120.6^{\mathrm{b}}$ & $1034.2 \pm 112.6^{\mathrm{b}}$ \\
Rice & $5.44 \pm 0.43^{\mathrm{c}}$ & $851.0 \pm 103.2^{\mathrm{ab}}$ & $713.4 \pm 87.4^{\mathrm{a}}$ \\
Wheat & $0.35 \pm 0.14^{\mathrm{a}}$ & $730.4 \pm 153.6^{\mathrm{a}}$ & $24.4 \pm 1.2^{\mathrm{a}}$ \\
\hline
\end{tabular}

The data is presented as the mean $\pm S D(n=3)$

a,b Different letters indicate significantly different values according to a one-way ANOVA with Duncan's multiple test $(p<0.05)$ 
perform significantly difference on the concentration of polysaccharide produced by $C$. cicadae NTTU 868 fruit bodies $(p>0.05)$. As a result, the oat was regarded as the optimal substrate to produce higher adenosine and HEA concentrations of C. cicadae NTTU 868 fruit bodies.

\section{Growth of submerged-fermented C. cicadae NTTU 868 mycelium and the change of adenosine and HEA concentration}

We also investigated the growth curve of C. cicadae NTTU 868 mycelium. The growth curve is shown in Fig. 2a. The biomass can be rapidly increased before Day 2. The growth curve of biomass reached the stationary phase from Day 2 to Day 10. As shown in Fig. 2b, higher adenosine and HEA concentrations were observed on Day 2 and Day 10. The reason may be that the adenosine, a purine nucleoside, is an important material for the biomass growth and metabolism. However, adenosine and HEA concentrations decreased dramatically from Day 2 to Day 6, and then increased until Day 10. Furthermore, the fermentation capacity was scaled up in order to investigate whether adenosine, HEA and polysaccharide concentrations were stable using $1000-\mathrm{mL}$ scale of submerged fermentation. As shown in Fig. 3, C. cicadae NTTU 868 mycelium had significantly higher adenosine and HEA concentrations on Day 4. However, adenosine and HEA concentrations decreased dramatically from Day 6 and then it slightly increased after Day 10. The production curve of polysaccharide in $1000-\mathrm{mL}$ scale was similar to that in $100-\mathrm{mL}$ scale, the polysaccharide production was increased with the increasing culture time. a

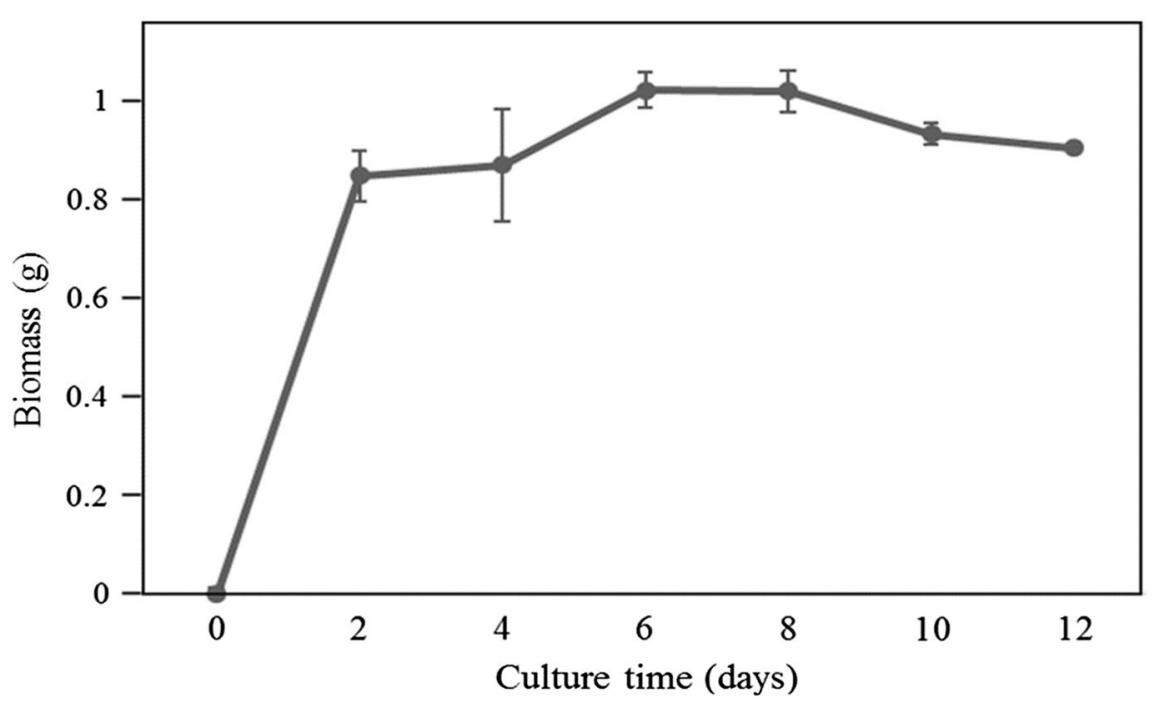

b

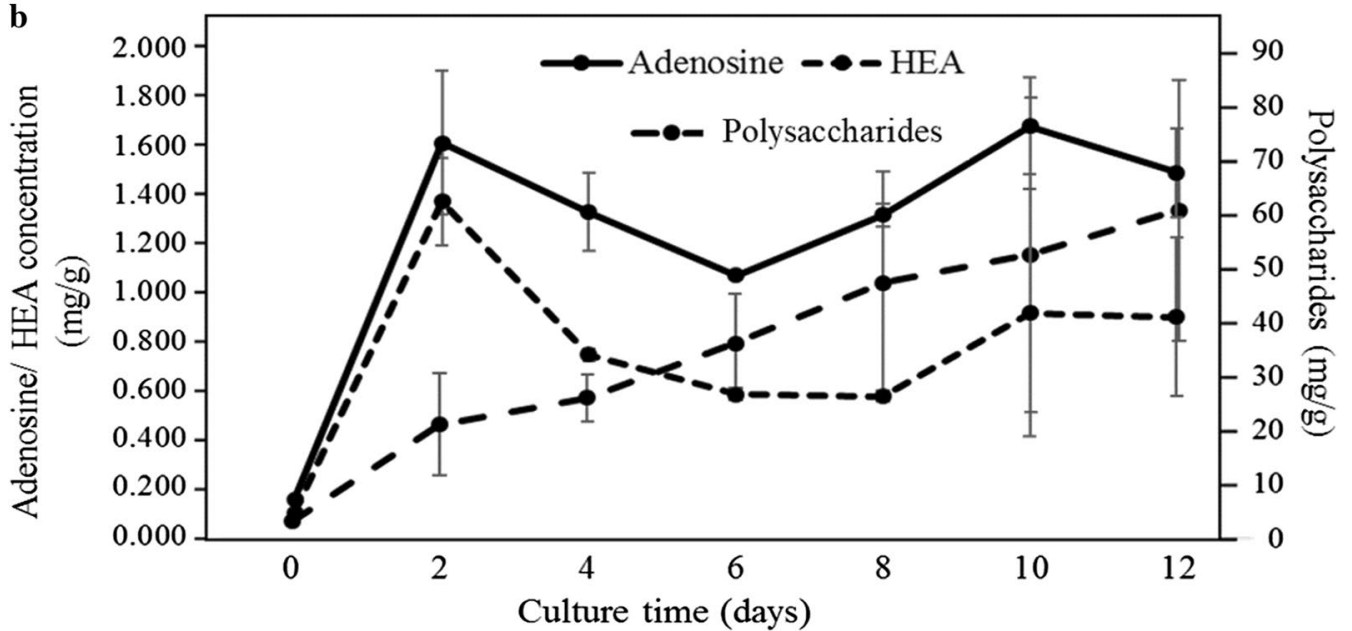

Fig. 2 Growth curve of submerged-fermented C. cicadae NTTU 868 mycelium and the change of adenosine and HEA concentration: a dry mycelium biomass; $\mathbf{b}$ adenosine, HEA and polysaccharide concentration by using HPLC analysis. The data is presented as the mean $\pm S D(n=3)$ 


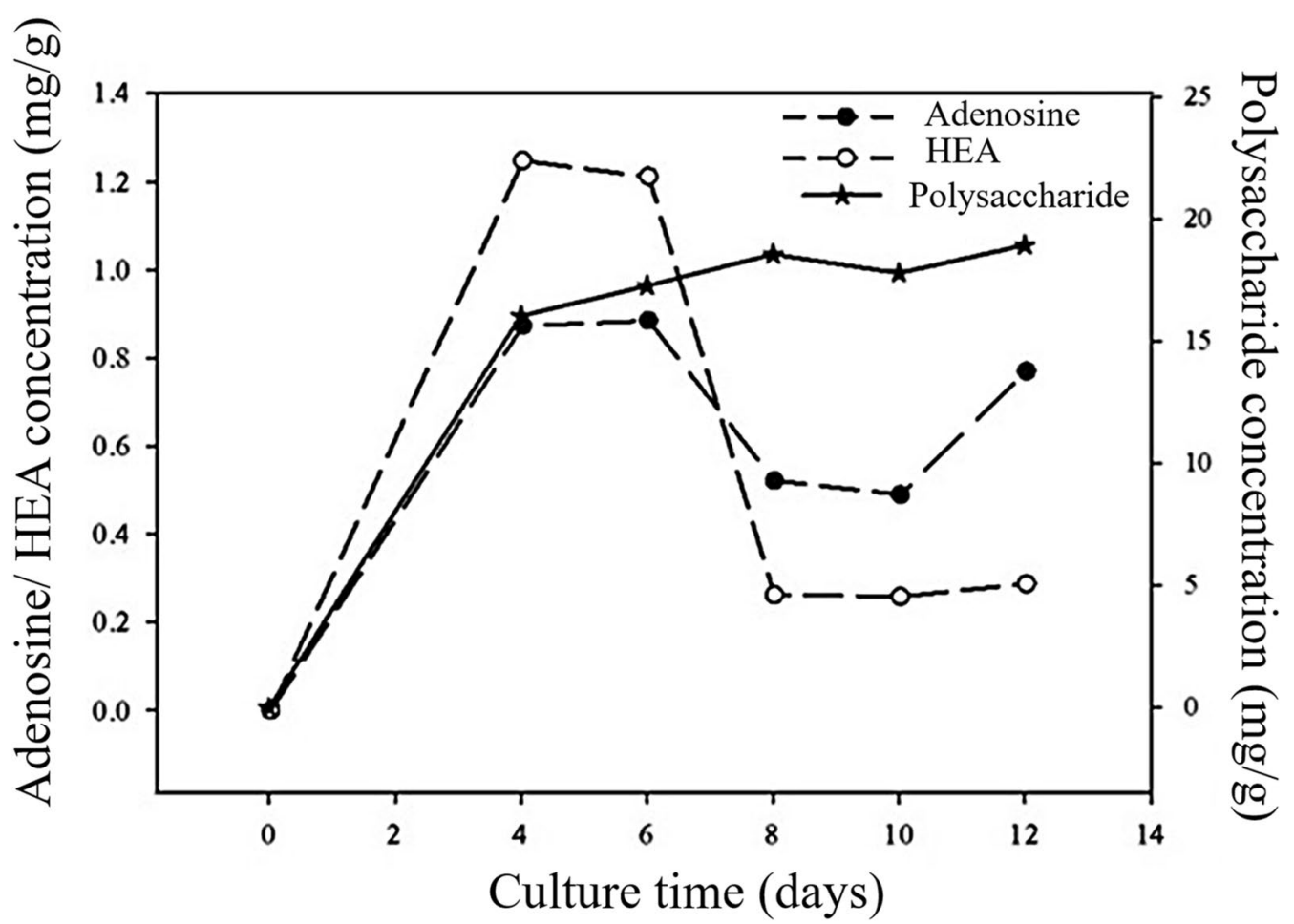

Fig. 3 Time course of adenosine, HEA and polysaccharide contents under pilot-up fermentation of C. cicadae NTTU 868

Effect of different basal mediums on adenosine, HEA and polysaccharide in $100-\mathrm{mL}$ submerged-fermented $C$. cicadae NTTU868 mycelium

In order to investigate the culture condition of $C$. cicadae NTTU 868 mycelium, various grain powders such as the barley, oat, and rice powder (2\%) were used as the culture substrate to produce mycelium respectively. As shown in Table 2, adenosine concentrations produced under barley, oat, and rice substrates on Day 10 were significantly higher than that on Day $2(p<0.05)$. The increased trend was also found on HEA concentrations produced under barley and oat substrates. However, we also compared adenosine and HEA concentrations of fermented products between grain powders and PDB. PDB had significantly greater adenosine and HEA concentrations than the other grain powders $(p<0.05)$. Furthermore, HEA concentration had reached the highest level on Day 2, which indicated that submerged culture with PDB can be used as a rapid fermentation mode for HEA production of C. cicadae.

Effect of carbon, nitrogen and mineral supplement on adenosine, HEA and polysaccharide contents of $100-\mathrm{mL}$ submerged-fermented $C$. cicadae NTTU 868 mycelium

To optimize the submerged culture condition of $C$. cicadae NTTU 868, 0.2\% of carbon sources (dextrose,

Table 2 Effect of different basal mediums on adenosine, HEA and polysaccharide in 100-mL submerged-fermented C. cicadae NTTU868 mycelium

\begin{tabular}{|c|c|c|c|c|}
\hline \multirow[t]{2}{*}{ Substrates } & \multicolumn{2}{|c|}{ Adenosine (mg/kg biomass) } & \multicolumn{2}{|c|}{ HEA (mg/kg biomass) } \\
\hline & Day 2 & Day 10 & Day 2 & Day 10 \\
\hline Barley & $75.8 \pm 41.2^{\mathrm{a}}$ & $442.4 \pm 16.4^{b}$ & $45.1 \pm 13.0^{\mathrm{a}}$ & $114.0 \pm 6.6^{b}$ \\
\hline Oat & $102.9 \pm 44.9^{\mathrm{a}}$ & $529.3 \pm 79.3^{b}$ & $3.9 \pm 1.6^{\mathrm{a}}$ & $102.8 \pm 11.7^{b}$ \\
\hline Rice & $17.5 \pm 12.3^{\mathrm{a}}$ & $225.5 \pm 52.7^{b}$ & $1.2 \pm 3.0^{\mathrm{a}}$ & $9.2 \pm 7.8^{\mathrm{a}}$ \\
\hline PDB & $1601.6 \pm 295.7^{\mathrm{a}}$ & $1669.3 \pm 199.0^{\mathrm{a}}$ & $1359.5 \pm 176.8^{\mathrm{a}}$ & $903.6 \pm 509.2^{1}$ \\
\hline
\end{tabular}

The data is presented as the mean $\pm S D(n=3)$

a,b Different letters indicate significantly different values according to a one-way ANOVA with Duncan's multiple test $(p<0.05)$ 
mannitol, or sucrose), $0.2 \%$ of nitrogen sources (monosodium glutamate, peptone or yeast extract) and $0.05 \%$ of mineral supplement (potassium chloride, magnesium chloride or ferric chloride) were added into the PDB, respectively. The result in Table 3 indicated that all of the additional carbon sources (dextrose, mannitol, sucrose) in the PDB performed no significant result on adenosine, HEA, and polysaccharide concentrations among each group $(p>0.05)$. Using peptone and yeast extract as additional sources of nitrogen source produced higher adenosine concentration than using MSG $(p<0.05)$. However, all of the additional nitrogen sources had no significant effect on HEA and polysaccharide concentration among each group $(p>0.05)$. The addition of ferric chloride may inhibit the growth of $C$. cicadae NTTU 868, resulting in significantly lower concentrations of adenosine, HEA and polysaccharide than potassium chloride and magnesium chloride $(p<0.05)$. The additional potassium chloride and magnesium chloride in the PDB was not able to increase adenosine concentration $(p>0.05)$. However, additional potassium chloride in the PDB contained higher concentrations of HEA than in magnesium chloride in the PDB $(p<0.05)$.

Effect of different concentration of dextrose, yeast extract and $\mathrm{KCl}$ on adenosine, HEA and polysaccharide in $100-\mathrm{mL}$ submerged-fermented $C$. cicadae NTTU868 mycelium

An additional $0.2 \%$ of dextrose in the PDB resulted in the C. cicadae NTTU 868 mycelium having higher adenosine

Table 3 Effect of carbon, nitrogen and mineral supplement on adenosine, HEA and polysaccharide contents of $100-\mathrm{mL}$ submerged-fermented C. cicadae NTTU868 mycelium

\begin{tabular}{llll}
\hline Substrates & $\begin{array}{l}\text { Adenosine } \\
\text { (mg/kg } \\
\text { biomass) }\end{array}$ & $\begin{array}{l}\text { HEA (mg/kg } \\
\text { biomass) }\end{array}$ & $\begin{array}{l}\text { Polysaccharide } \\
\text { (g/kg biomass) }\end{array}$ \\
\hline
\end{tabular}

Extra carbon sources

\begin{tabular}{lccc} 
Dextrose & $1095 \pm 16^{\mathrm{a}}$ & $473 \pm 7^{\mathrm{a}}$ & $16.2 \pm 0.9^{\mathrm{a}}$ \\
Mannitol & $884 \pm 356^{\mathrm{a}}$ & $425 \pm 57^{\mathrm{a}}$ & $15.6 \pm 0.8^{\mathrm{a}}$ \\
Sucrose & $964 \pm 274^{\mathrm{a}}$ & $386 \pm 82^{\mathrm{b}}$ & $15.9 \pm 0.9^{\mathrm{a}}$ \\
$\begin{array}{lc}\text { Extra nitrogen sources } \\
\text { MSG }\end{array}$ & $607 \pm 46^{\mathrm{a}}$ & $1335 \pm 1230^{\mathrm{a}}$ & $15.9 \pm 1.7^{\mathrm{a}}$ \\
Peptone & $1093 \pm 22^{\mathrm{b}}$ & $770 \pm 57^{\mathrm{a}}$ & $17.6 \pm 1.6^{\mathrm{a}}$ \\
Yeast extract & $1266 \pm 339^{\mathrm{b}}$ & $1171 \pm 1315^{\mathrm{a}}$ & $16.8 \pm 1.1^{\mathrm{a}}$ \\
Extra mineral supplement & & \\
$\mathrm{KCl}$ & $1038 \pm 49^{\mathrm{b}}$ & $481 \pm 118^{\mathrm{c}}$ & $16.4 \pm 0.7^{\mathrm{b}}$ \\
$\mathrm{MgCl}_{2}$ & $960 \pm 143^{\mathrm{b}}$ & $279 \pm 6^{\mathrm{b}}$ & $15.9 \pm 0.6^{\mathrm{ab}}$ \\
$\mathrm{FeCl}_{3}$ & $344 \pm 57^{\mathrm{a}}$ & $109 \pm 5^{\mathrm{a}}$ & $15.0 \pm 0.4^{\mathrm{a}}$ \\
\hline
\end{tabular}

The data is presented as the mean $\pm S D(n=3)$

MSG monosodium glutamate, $\mathrm{KCl}$ potassium chloride, $\mathrm{MgCl}_{2}$ magnesium chloride, $\mathrm{FeCl}_{3}$ ferric chloride

$a, b$ Different letters indicate significantly different values according to a one-way ANOVA with Duncan's multiple test $(p<0.05)$ and HEA content. We researched different concentrations $(0 \%, 0.04 \%, 0.2 \%$ or $1.0 \%)$ of dextrose in the PDB to investigate the effect on the production of adenosine, HEA, and polysaccharide in the $C$. cicadae NTTU 868 mycelium. In Table 4, the culture medium without additional dextrose produced the highest HEA and polysaccharide concentrations in the $C$. cicadae NTTU 868 mycelium $(p<0.05)$. Therefore, additional dextrose was not able to increase the production of HEA and polysaccharide.

According to Table 3 , an additional $0.2 \%$ yeast extract in the PDB resulted in higher adenosine production in C. cicadae NTTU 868 mycelium. We compared different concentrations $(0 \%, 0.04 \%, 0.2 \%$ or $1.0 \%)$ of yeast extract in the PDB and conducted research to find the best recipe for the culture medium. The result indicated that $0.2 \%$ of yeast extract in the PDB significantly increased the HEA production in C. cicadae NTTU 868 mycelium $(p<0.05)$. However, the productions of adenosine and polysaccharide in the C. cicadae NTTU 868 mycelium showed no significantly difference $(p>0.05)$ as using various concentrations of additional yeast extract.

Furthermore, an additional $0.05 \%$ of potassium chloride in the PDB produced higher levels of adenosine,

Table 4 Effect of different concentration of dextrose, yeast extract and $\mathrm{KCl}$ on adenosine, HEA and polysaccharide in $100-\mathrm{mL}$ submerged-fermented C. cicadae NTTU868 mycelium

\begin{tabular}{|c|c|c|c|}
\hline Sources & $\begin{array}{l}\text { Adenosine } \\
\text { (mg/kg } \\
\text { biomass) }\end{array}$ & HEA (mg/kg biomass) & $\begin{array}{l}\text { Polysaccharide } \\
\text { (g/kg biomass) }\end{array}$ \\
\hline \multicolumn{4}{|c|}{ Extra dextrose (\%) } \\
\hline 0.00 & $1080 \pm 56^{b}$ & $533 \pm 33^{c}$ & $20.5 \pm 1.1^{b}$ \\
\hline 0.04 & $1020 \pm 56^{b}$ & $472 \pm 15^{b c}$ & $18.9 \pm 2.0^{\mathrm{ab}}$ \\
\hline 0.20 & $964 \pm 90^{b}$ & $432 \pm 59^{b}$ & $17.6 \pm 0.2^{a}$ \\
\hline 1.00 & $656 \pm 125^{b}$ & $337 \pm 24^{a}$ & $19.4 \pm 0.9^{a b}$ \\
\hline \multicolumn{4}{|c|}{ Extra yeast extract (\%) } \\
\hline 0.00 & $1080 \pm 56^{a}$ & $533 \pm 33^{\mathrm{a}}$ & $20.5 \pm 1.1^{a}$ \\
\hline 0.04 & $1134 \pm 81^{a}$ & $544 \pm 38^{a}$ & $21.3 \pm 4.0^{\mathrm{a}}$ \\
\hline 0.20 & $1157 \pm 376^{\mathrm{a}}$ & $764 \pm 120^{b}$ & $20.8 \pm 2.5^{a}$ \\
\hline 1.00 & $1169 \pm 22^{a}$ & $634 \pm 137^{a b}$ & $22.4 \pm 6.1^{\mathrm{a}}$ \\
\hline \multicolumn{4}{|c|}{ Extra KCl (\%) } \\
\hline 0.00 & $1080 \pm 56^{c}$ & $533 \pm 33^{b}$ & $20.5 \pm 1.1^{\mathrm{a}}$ \\
\hline 0.01 & $1035 \pm 60^{c}$ & $479 \pm 40^{b}$ & $21.2 \pm 1.0^{\mathrm{a}}$ \\
\hline 0.05 & $841 \pm 38^{b}$ & $474 \pm 81^{b}$ & $20.0 \pm 1.6^{\mathrm{a}}$ \\
\hline 0.25 & $670 \pm 56^{a}$ & $356 \pm 51^{a}$ & $21.6 \pm 0.7^{a}$ \\
\hline
\end{tabular}

The data is presented as the mean $\pm S D(n=3)$

$\mathrm{KCl}$ potassium chloride

$a, b, c$ Different letters indicate significantly different values according to a oneway ANOVA with Duncan's multiple test $(p<0.05)$ 
HEA and polysaccharide in the C. cicadae NTTU 868 mycelium than when magnesium chloride and ferric chloride were used. However, lower concentrations of potassium chloride in the PDB produced significantly higher levels of adenosine and HEA in the C. cicadae NTTU 868 mycelium $(p<0.05)$. The levels of polysaccharide in the $C$. cicadae NTTU 868 mycelium had no significant change as using various concentrations $(0 \%$, $0.01 \%, 0.05 \%$ or $0.25 \%$ ) of additional potassium chloride $(p>0.05)$.

\section{The difference of adenosine, HEA and polysaccharide production of $C$. cicadae NTTU 868 mycelium between different cultivation volume submerged fermentation}

In the end, we compared the levels of adenosine, HEA and polysaccharide in two different culture scale (100$\mathrm{mL}$ or $1500-\mathrm{mL}$ ) of cultured C. cicadae NTTU 868 mycelium. The result (Table 5) indicated that 100$\mathrm{mL}$ of culture scale (with an additional $0.2 \%$ of yeast extract in the $\mathrm{PDB}$ ) produced significantly higher levels of adenosine and polysaccharide than $1500-\mathrm{mL}$ of the culture scale $(p<0.05)$. In contrast, $1500-\mathrm{mL}$ of culture scale produced significantly higher levels of HEA than $100 \mathrm{~mL}$ of culture scale $(p<0.05)$. Therefore, submerged culture with PDB medium including additional $0.2 \%$ of yeast extract was proven to perform a rapid and high productivity culture mode for scale-up production of the functional compound HEA of C. cicadae NTTU 868.

\section{Discussion}

The wild $C$. cicadae fruit bodies usually grow in high humidity conditions on mountains between 80 and $500 \mathrm{~m}$ high. Because wild C. cicadae fruit bodies grow slowly and are easily infected by other moulds, the artificially-cultured C. cicadae fruit bodies have higher safety and more efficient to produce. In this study, C. cicadae NTTU 868 was a high production strain for HEA and polysaccharide productions. This study researched optimal solid fermentation conditions in order to develop

\begin{tabular}{|c|c|c|c|}
\hline $\begin{array}{l}\text { Culture } \\
\text { scale } \\
(\mathrm{mL})\end{array}$ & $\begin{array}{l}\text { Adenosine } \\
\text { (mg/kg } \\
\text { biomass) }\end{array}$ & HEA (mg/kg biomass) & $\begin{array}{l}\text { Polysaccharide } \\
\text { (g/kg biomass) }\end{array}$ \\
\hline 100 & $1157 \pm 376$ & $764 \pm 120$ & $20.7 \pm 2.5$ \\
\hline 1500 & $240 \pm 31$ & $1157 \pm 449$ & $14.3 \pm 0.4$ \\
\hline
\end{tabular}

the artificial culture technology. In previous research, it was shown that artificial-cultured $C$. cicadae fruit bodies grew in silkworms (Hu et al. 2009). The success rate of artificial-cultured C. cicadae fruit bodies is almost $100 \%$ using silkworm pupae, and about 70\% using silkworm larvae. Another C. cicadae strain, C. cicadae MP12 was collected from the Shennongjia Mountains in mainland China. The artificial-cultured C. cicadae MP12 fruit bodies were successfully grown on Cryptotympana atrata pupae and a rice base medium (Wang et al. 2012). However, these materials are not easy to get, which will limit the scale-up production in the industrial development. In this study, we used barley, oat, rice and wheat as grain substrates to culture C. cicadae NTTU 868 fruit bodies. Using oat as the substrate was able to produce the highest content of fruit bodies than using the other substrates. In general, rice and wheat were used as the main grain substrates for the culture of Cordyceps species. However, $C$. cicadae NTTU 868 fruit bodies produced the lowest contents of HEA under the rice substrate. This study found that oat was the optimal solid substrate rather than wheat for the productions of fruit bodies, adenosine, and HEA. However, the culture time of solid fermentation was still over than 60 days.

Fermentation time and scale-up were both regarded as the important limitation factors for the development of fungi industry. Submerged culture is easy to scale-up, because it can be carried out by automatic production to achieve concatenation work, save time and improve efficiency. Therefore, this study further investigated the submerged culture of $C$. cicadae NTTU 868 mycelium. Mycelium produced by submerged culture and fruit bodies by solid culture had similar amounts of adenosine and HEA. However, the time of submerged culture was less than 10 days. Submerged fermentation produced the highest amounts of adenosine and HEA in $C$. cicadae NTTU 868 mycelium on day 2 and day 10. It also produced higher biomass of C. cicadae NTTU 868 mycelium. Therefore, submerged fermentation of C. cicadae NTTU 868 can easy scale up in the fermentation industry.

Although submerged culture was suitable technology to produce mycelium of $C$. cicadae, the culture medium should be a key factor for high production of HEA. Therefore, the previous studies also studied the effect of the medium compositions containing different carbon sources, nitrogen sources and mineral supplements on the growth and the production of bioactive compounds in C. cicadae. However, the previous study on the production of HEA was still rare, but we can understand the effect of medium composition in the previous studies of polysaccharides fermentation of $C$. cicadae or submerged fermentation of other Cordyceps species (Sharma

The data is presented as the mean $\pm S D(n=3)$ 
et al. 2015a, b). The carbon sources including mannitol, sucrose and glucose increased the extracellular polysaccharide (EPS) and intracellular polysaccharide (IPS) production in the submerged culture of $C$. cicadae significantly $(p<0.05)$. Previous researches indicated that dextrose is the best carbon source for Cordyceps species (Sharma et al. 2015a, b). In this study, adding 0.2\% by volume quantity of dextrose in the PDB resulted in higher concentrations of adenosine and HEA in the $C$. cicadae NTTU 868 mycelium than using mannitol or sucrose. In the previous study, yeast extract and peptone both produced higher amounts of EPS and IPS in the C. cicadae mycelium than other nitrogen sources (Sharma et al. 2015b). In this study, $0.2 \%$ of yeast extract was still the optimal additional nitrogen source in the PDB for increasing the concentration of adenosine and HEA. Above results were also similar to the results of other studies on another C. cicadae strain and C. sinensis (Dong and Yao 2005). Therefore, yeast extract was a well nitrogen source for the culture of many Cordyceps species as well as the production of HEA of C. cicadae. Ions and salts also affected the growth and metabolism of fungi. The mineral sources, monopotassium phosphate, cobalt(II) chloride hexahydrate and iron(II) sulfate heptahydrate, in the culture medium increased the concentrations of EPS and IPS in the C. cicadae mycelium (Sharma et al. 2015b). Potassium chloride has been observed as being the optimal mineral source to produce higher concentrations of adenosine in C. militaris (Hung et al. 2015) and higher concentration of EPS and IPS in another C. cicadae strain and C. sinensis (Dong and Yao 2005; Sharma et al. 2015a). This study also observed that additional potassium chloride in PDB was able to produce higher productions of adenosine and HEA in the $C$. cicadae NTTU 868 mycelium.

Adenosine, HEA and polysaccharide performed different production curves during the fermentation of $C$. cicadae NTTU 868. Furthermore, C. cicadae NTTU 868 performed different growth curve in $100-\mathrm{mL}$ and $1500-$ $\mathrm{mL}$ fermentation scales. The highest levels of concentration of adenosine and HEA were observed on Day 2 and Day 4 in 100-mL and 1500-mL fermentation scale, respectively. Polysaccharide production can be increased with the fermentation time in $100-\mathrm{mL}$ or $1500-\mathrm{mL}$ fermentation scales. However, Day 4-6 can be regarded as the optimal fermentation time for the production of adenosine, HEA and polysaccharide in the $1500-\mathrm{mL}$ submerged fermentation scale. In conclusion, solid and submerged fermentation can produce similar concentrations of adenosine, HEA and polysaccharide. Adding $0.2 \%$ of yeast extract in the PDB, at $24{ }^{\circ} \mathrm{C}$ for 10 days created the most suitable fermentation condition for the C. cicadae NTTU 868 mycelium. However, submerged fermentation is more efficient for adenosine and HEA production than using solid-fermentation. Importantly, it can be easily scaled-up in the fermentation industry to develop medical potential in the industry.

\section{Acknowledgements \\ Not applicable.}

Authors' contributions

Both C-LL and B-JK read and approved the final manuscript.

Funding

Not applicable.

Availability of data and materials

Not applicable.

Ethics approval and consent to participate

This article does not contain any studies with human participants or animals performed by any of the authors.

\section{Consent for publication}

The authors consent the publication in AMB express.

Competing interests

The authors declare that they have no competing interests.

Received: 1 August 2018 Accepted: 5 October 2019

Published online: 09 December 2019

\section{References}

Dong CH, Yao YJ (2005) Nutritional requirements of mycelial growth of Cordyceps sinensis in submerged culture. J Appl Microbiol 99(3):483-492. https://doi.org/10.1111/j.1365-2672.2005.02640.x

Hu H, Zou X, Luo L, Liu A, Liang Z (2009) Artificial culturing of Cordyceps cicadidae on living silkworm. Zhongguo Zhong Yao Za Zhi 34(17):2140-2143

Hung YP, Wang JJ, Wei BL, Lee CL (2015) Effect of the salts of deep ocean water on the production of cordycepin and adenosine of Cordyceps militarisfermented product. AMB Express 5(1):140. https://doi.org/10.1186/s1356 8-015-0140-5

Ke BJ, Lee CL (2018) Cordyceps cicadae NTTU 868 mycelium prevents CCl4induced hepatic fibrosis in BALB/C mice via inhibiting the expression of pro-inflammatory and pro-fibrotic cytokines. J Func Food 43:214-223

Koupenova M, Ravid K (2013) Adenosine, adenosine receptors and their role in glucose homeostasis and lipid metabolism. J Cell Physiol. https://doi. org/10.1002/jcp.24352

Lee H, Kim YJ, Kim HW, Lee DH, Sung MK, ParkT (2006) Induction of apoptosis by Cordyceps militaris through activation of caspase-3 in leukemia HL-60 cells. Biol Pharm Bull 29(4):670-674

Lu MY, Chen CC, Lee LY, Lin TW, Kuo CF (2015) N(6)-(2-Hydroxyethyl)adenosine in the medicinal mushroom Cordyceps cicadae attenuates lipopolysaccharide-stimulated pro-inflammatory responses by suppressing TLR4mediated NF-kappaB signaling pathways. J Nat Prod 78(10):2452-2460. https://doi.org/10.1021/acs.jnatprod.5b00573

Meng Z, Kang J, Wen T, Lei B, Hyde KD (2015) Cordycepin and N6-(2-hydroxyethyl)-adenosine from Cordyceps pruinosa and their interaction with human serum albumin. PLoS ONE 10(3):e0121669. https://doi. org/10.1371/journal.pone.0121669

Park C, Hong SH, Lee JY, Kim GY, Choi BT, Lee YT, Park DI, Park YM, Jeong YK, Choi YH (2005) Growth inhibition of U937 leukemia cells by aqueous extract of Cordyceps militaris through induction of apoptosis. Oncol Rep 13(6):1211-1216

Peng JH, Li XM, Hu YY, Feng Q (2013) Effect of Cordyceps polysaccharide on lipid peroxidation of rats with dimethylnitrosamine-induced liver fibrosis. Zhongguo Zhong Yao Za Zhi 38(3):391-396

Ramesh T, Yoo SK, Kim SW, Hwang SY, Sohn SH, Kim IW, Kim SK (2012) Cordycepin (3'-deoxyadenosine) attenuates age-related oxidative stress 
and ameliorates antioxidant capacity in rats. Exp Gerontol 47(12):979987. https://doi.org/10.1016/j.exger.2012.09.003

Sharma SK, Gautam N, Atri NS (2015a) Optimization, composition, and antioxidant activities of exo- and intracellular polysaccharides in submerged culture of Cordyceps gracilis (Grev.) Durieu \& Mont. Evid Based Complement Alternat Med 462:864. https://doi.org/10.1155/2015/462864

Sharma SK, Gautam N, Atri NS (2015b) Optimized extraction, composition, antioxidant and antimicrobial activities of exo and intracellular polysaccharides from submerged culture of Cordyceps cicadae. BMC Complement Altern Med 15:446. https://doi.org/10.1186/s12906-015-0967-y

Sharma SK, Gautam N, Atri NS (2018) Retraction Note: Optimized extraction, composition, antioxidant and antimicrobial activities of exo and intracellular polysaccharides from submerged culture of Cordyceps cicadae. BMC Complement Altern Med 18(1):276. https://doi.org/10.1186/s1290 6-018-2344-0

Sohn SH, Lee SC, Hwang SY, Kim SW, Kim IW, Ye MB, Kim SK (2012) Effect of long-term administration of cordycepin from Cordyceps militaris on testicular function in middle-aged rats. Planta Med 78(15):1620-1625. https ://doi.org/10.1055/s-0032-1315212

Wang Y, Guo Y, Zhang L, Wu J (2012) Characterizations of a new Cordyceps cicadae isolate and production of adenosine and cordycepin. Braz J Microbiol 43(2):449-455. https://doi.org/10.1590/S1517-83822012000200004

Wang J, Zhang DM, Jia JF, Peng QL, Tian HY, Wang L, Ye WC (2014) Cyclodepsipeptides from the ascocarps and insect-body portions of fungus Cordyceps cicadae. Fitoterapia 97:23-27

Weng SC, Chou CJ, Lin LC, Tsai WJ, Kuo YC (2002) Immunomodulatory functions of extracts from the Chinese medicinal fungus Cordyceps cicadae. Ethnopharmacol 83(1-2):79-85
Won SY, Park EH (2005) Anti-inflammatory and related pharmacological activities of cultured mycelia and fruiting bodies of Cordyceps militaris. J Ethnopharmacol 96(3):555-561. https://doi.org/10.1016/j.jep.2004.10.009

Yu HM, Wang BS, Huang SC, Duh PD (2006) Comparison of protective effects between cultured Cordyceps militaris and natural Cordyceps sinensis against oxidative damage. J Agric Food Chem 54(8):3132-3138. https:// doi.org/10.1021/jf053111w

Zeng WB, Yu H, Ge F, Yang JY, Chen ZH, Wang YB, Dai YD, Adams A (2014) Distribution of nucleosides in populations of Cordyceps cicadae. Molecules 19(5):6123-6141. https://doi.org/10.3390/molecules19056123

Zhou X, Luo L, Dressel W, Shadier G, Krumbiegel D, Schmidtke P, Zepp F, Meyer CU (2008) Cordycepin is an immunoregulatory active ingredient of Cordyceps sinensis. Am J Chin Med 36(5):967-980. https://doi. org/10.1142/S0192415X08006387

Zhu R, Zheng R, Deng Y, Chen Y, Zhang S (2014) Ergosterol peroxide from Cordyceps cicadae ameliorates TGF-beta1-induced activation of kidney fibroblasts. Phytomedicine 21(3):372-378. https://doi.org/10.1016/j. phymed.2013.08.022

\section{Publisher's Note}

Springer Nature remains neutral with regard to jurisdictional claims in published maps and institutional affiliations.

\section{Submit your manuscript to a SpringerOpen ${ }^{\circ}$ journal and benefit from:}

- Convenient online submission

- Rigorous peer review

- Open access: articles freely available online

- High visibility within the field

- Retaining the copyright to your article

Submit your next manuscript at $\boldsymbol{\nabla}$ springeropen.com 